\title{
Information Centre by Virtual Computer Through a Multi-Technology Framework
}

\author{
Pedro Ramos Brandao \\ Department of Interdisciplinary Center for History, Cultures and Societies (CIDEHUS), University of Évora, Évora, Portugal \\ Email address: \\ pb@pbrandao.net \\ To cite this article: \\ Pedro Ramos Brandao. Information Centre by Virtual Computer Through a Multi-Technology Framework. Internet of Things and Cloud \\ Computing. Vol. 5, No. 4, 2017, pp. 71-75. doi: 10.11648/j.iotcc.20170504.12
}

Received: September 1, 2017; Accepted: December 15, 2017; Published: January 4, 2018

\begin{abstract}
Development of a framework based in virtualization to be implemented in an information centre/ library (IC/LIB). This framework combines three technologies: virtualization, SQL, ADO.Net. It will be implemented in libraries and information centres to allow users/researchers to keep and synchronize the information with other devices and cloud computing. The individual virtual machine maintains all users' work information after the end of the work session. However, the work information is accessible by synchronization with a cloud computer platform. Hyper-V 2012 R2 will support this framework.
\end{abstract}

Keywords: Virtualization, Library, SQL, Hyper-V

\section{Introduction}

The main purpose of this study is to propose the creation of a model of the Framework type which facilitates and modernize user access instrumentation of information centres to information itself, as making non-volatile the collected data keeping it in the structure of the information centre. This model has, as main base virtualization, at a technological level. By providing each user an individual virtual machine, he may easily and faster gain access to all the information he is entitled, which will be stored at a SQL Virtual Server (whose data structure will be an integral part of this study), through a friendly interface developed in ADO.NET. The framework will comprise a safe solution for users to synchronize the data stored at their virtual machine with any other device outside the information centre.

\section{Development Approach}

\subsection{Information Centres and Libraries}

Most contemporary authors consider that information centres as well as libraries require a paradigm modification concerning their offer and interaction both with their users as well as with general public. Today's libraries and information centres may be considered obsolete with regard to information access type paradigm. [1]

There are many causes for this diagnose, one of them is the old-fashioned way user's community gain access to information, it may be considered-that if libraries are simple remediation instruments of mass access, an also obsolete issue due to the Internet technology [1], which paradoxically libraries and information centres do not use in its full available potential. There has been a decrease in the number of users of information centres, [1] in part due to the fact that one does not find within these institutions the technological tools they are used to use at home and at universities.

It is a matter of fact that many universities and information centres (from now called of IC) provide information through the web sites [1], however, this technology is extremely limited for what an investigator requires nowadays.

Some standards have already been set out with the aim to implement new technologies in the way IC function, that may be the conceptual basis for a future new use in operational models based on the latest information technologies [2]. However a significant part of the studies are based on technologies based on the HTTP (Hypertext Transfer Protocol) [2], which has, many limitations nowadays from safety to the defective frontline instrumentation of access to the database with connection and synchronization to Cloud and Computing Systems. These studies have one great merit, they lay out a topology of solutions which may be used in more sophisticated models of 
technological structures to access IC information. Namely, quality, performance as well as interconnectivity issues of profiles and knowledge bases. [2].

The issue of IC to grant users the access the information stored in their datacentres in a safe way whilst maintaining high levels of safety (which cannot be achieved with solutions such as HTTP or HTTPS), in which the paradigm of making a catalogue available reaches the level of providing an internal search engine, are also taken into account nowadays [3] “... They know that libraries could never contain it all within their Walls, because information is endless, extending everywhere on the Internet, and to find I tone needs a search engine, not a card catalogue..." [3], that is, conceptually a new structural model of access to information on IC shall go through sophisticated search engines and not by traditional cataloguing systems.

Within this framework, a new integrated solution may be presented, "... there is a future for public libraries in the Internet age...” [3], IC managers and user public would like that new information technologies will be introduced in the way IC operate and provide their data [3], we have once more found authors who diagnose, and well in our opinion, but do not provide the cure: "Ensuring even greater Access to computers for all. Almost two-thirds say having enough computers and online services for people who want to use them should be a high priority for libraries...”. [3], [4]

On the other hand, the creation of a framework, based on virtualization as well as in virtualized personal computers to IC users may accelerate another process, data digitalization, an unsurpassable issue, yet a slow process, nowadays but which must be carried out with priority: “... a very practical reason for digitizing books in electronic form, books aren't vulnerable to natural disasters or pulverization (...) as an important reminder of the vulnerability of cultural memory". [5]

David Lankes, in a presentation in 2008, at the University of Siracusa, develops a theory to the design of the new IC, in all their aspects. In his conclusion, he states that the digital era brings new conceptualizations. He states that IC shall make available a platform as catalogue, but in a multifaceted way, supported by differentiated information technologies, providing approaches that introduce the concepts of profiles and events related to contents. However, many of these proposals are based in solutions HTTP type. They propose the construction of a new platform for innovation within IC and Libraries. [1] Nonetheless, no technical solutions are provided as well as none of the technologies that shall be used are specified.

From this proposal, I intend to develop a model, based on virtualization to be applied in IC as well as libraries, providing a virtual computer to all authenticated users, from which is granted access to information using a specific connection interface to a centralised database. Universal database architecture is also an integral part of the final framework. Users can create communication tunnels between their virtual machine and cloud computing systems. Data resulting from IC investigation will be wherever the user is. (Figure 1) [8], [9], [10], [11], [12]

\subsection{Virtualization}

The concept of virtualization has been discussed since 1950, although it has become a trend with its implementation in the $x 86$ platform, in the 1980s. At the beginning of the 1960s, IBM has introduced the concept of "Time Sharing" (time-sharing), which has been the initial route for virtualization (through the concept of hardware sharing). [6]

In 1972, the System/370 Advanced Function was made available and had a new hardware of net addresses relocation as well as support to four new operating systems: VM/370, DOS/VS, OS/VS1 e OS/VS2. The introduction of the VM/370 Release 2, in 1974, contained the first interaction of micro code VMA (Virtual Machine Assist). In that same year, Gerald J. Popek e Robert P. Goldberg have created a set of formal requirements for architectures, whose greater relevance has been entitled "Formal Requirements for Virtualizable Third Generation Architectures". Whose greatest relevance was the conveniently presentation as well as easy to define in which circumstance a computer's architecture would effectively withstand virtualization, presenting the guidelines to the architectural design of computer's architectures intended to withstand. [7] 2011)

Its experience on MAC and PC platforms led a Connectix to create a product called Connectix Virtual PC 1.0. Virtual PC 1.0 was a real programming achievement, incorporating a binary translation mechanism for instructions of an Intel virtual x 86 processor, and for a physical PowerPC processor, used on MAC, thus allowing the virtualization of several operating systems, under machines which base operating system was MAC. This emulation example led Connectix to virtualization technology.

Chips manufacturers such as Intel or AMD continued to introduce new technologies in order to provide a better support to visualization on the hardware layer. These technologies include multi-core processors, Intel's virtualization technology (originally known as Vanderpool e Silvervale), e AMD-V/SVM (originally known as peaceful). These virtualization technologies on hardware level have allowed virtualization platforms to become more efficient, giving rise to the so-called virtualization Type One, in which MV could communicate directly to hardware easily. Intel-VT and AMD-V technologies have implemented these instructions and began to control the hypervisor so that there is no need of a software complex layer, that could give rise to performance issues, due to the non-correct communication of VM with hardware. Virtualization instructions added to AMD and Intel processors have helped to create new visualization platforms. [6] For example storing virtualization as well as intermediate network devices.

\subsection{The Framework}

The functioning of the final structure within the user context is as following: when the user access to IC he may use any available computer terminal, proceeding with his authentication through the Operating System, thereafter he requests a remote access session at which he will have to provide, once again, a 
username, a password as well as his virtual machine name. After that, he will gain access to his virtual computer.

There is as icon in his virtual computer workplace, which provides access to an interface that after executed makes a secure connection to the database in which data that specific IC provides is found. After collecting data, user may use a set of tools in order to edit, compile and store all the obtained data. The obtained data may be stored in his virtual computer, in order to be used in another session or to export to a cloud computing system outside IC so that after logging out IC, the user may access data from any other device.

Technically speaking the fundamentals of the framework to be developed will have the following characteristics: IC database will be fully centralised and managed by a 2014 SQL Server system, it shall be subject of an universal architecture which can adapt to any IC or libraries. This SQL system will be working in a virtual server, to allow high levels of redundancy, availability and easy recover in case of a disaster. These servers as well as all others within this network belong to a domain managed by an Active Directory made available by a 2012 R2 Windows Server Operating System. Remote connections from users to virtual computers are made available by the "Remote Desktop Connection" service (RDC). The connection and database access interface available on virtual machines is of the ADO.NET type.

When IC accredits a user, a virtual machine automatically produced from system shall be made available "Fabric" do System Centre Virtual Machine Manager 2014. The connection to cloud computing system by user from his virtual machine is performed trough HTTPS. (See Figure 2) [13], [14], [15], [16], [17], [18], [19], [20]

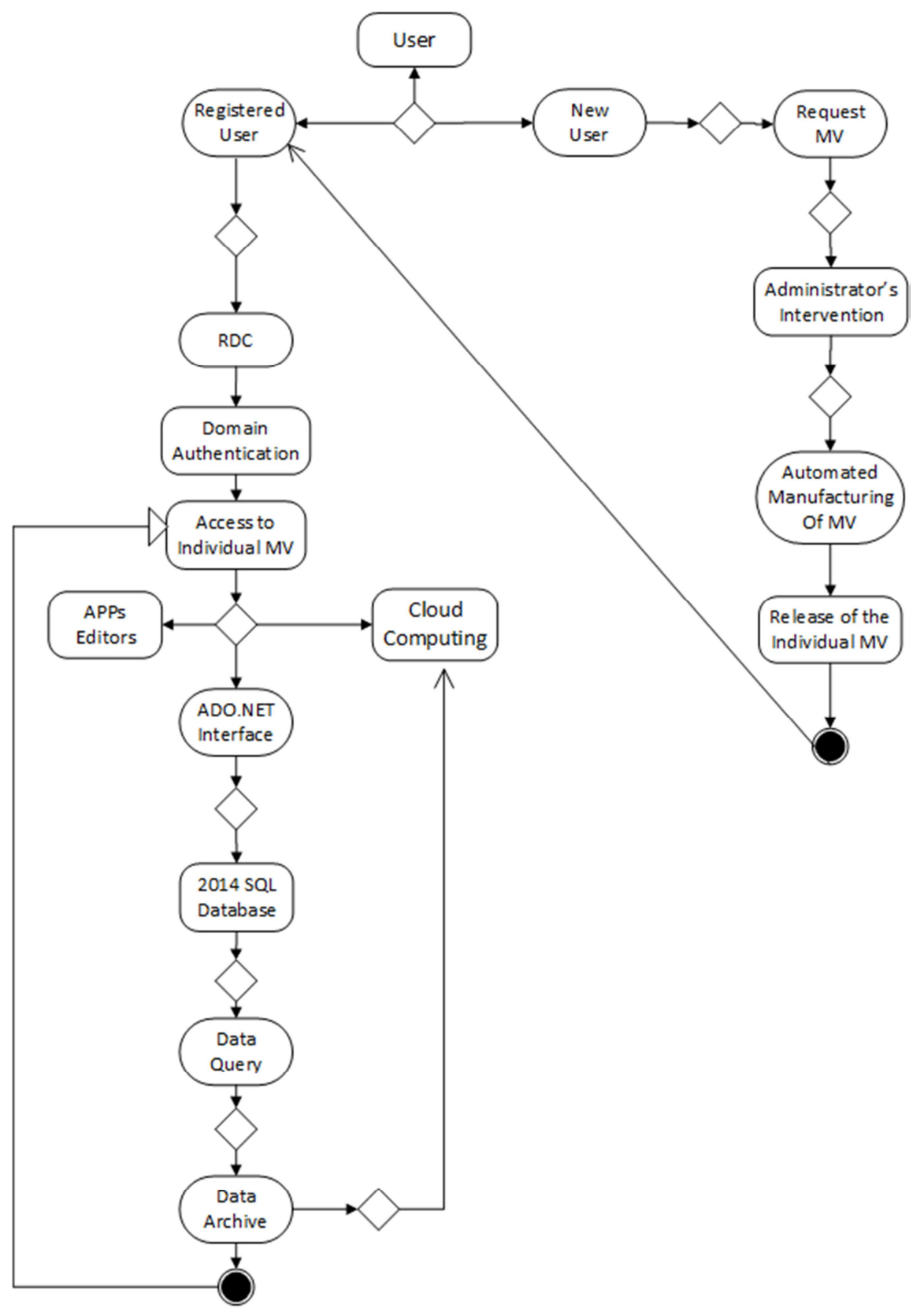

Figure 1. Framework Proposal. 


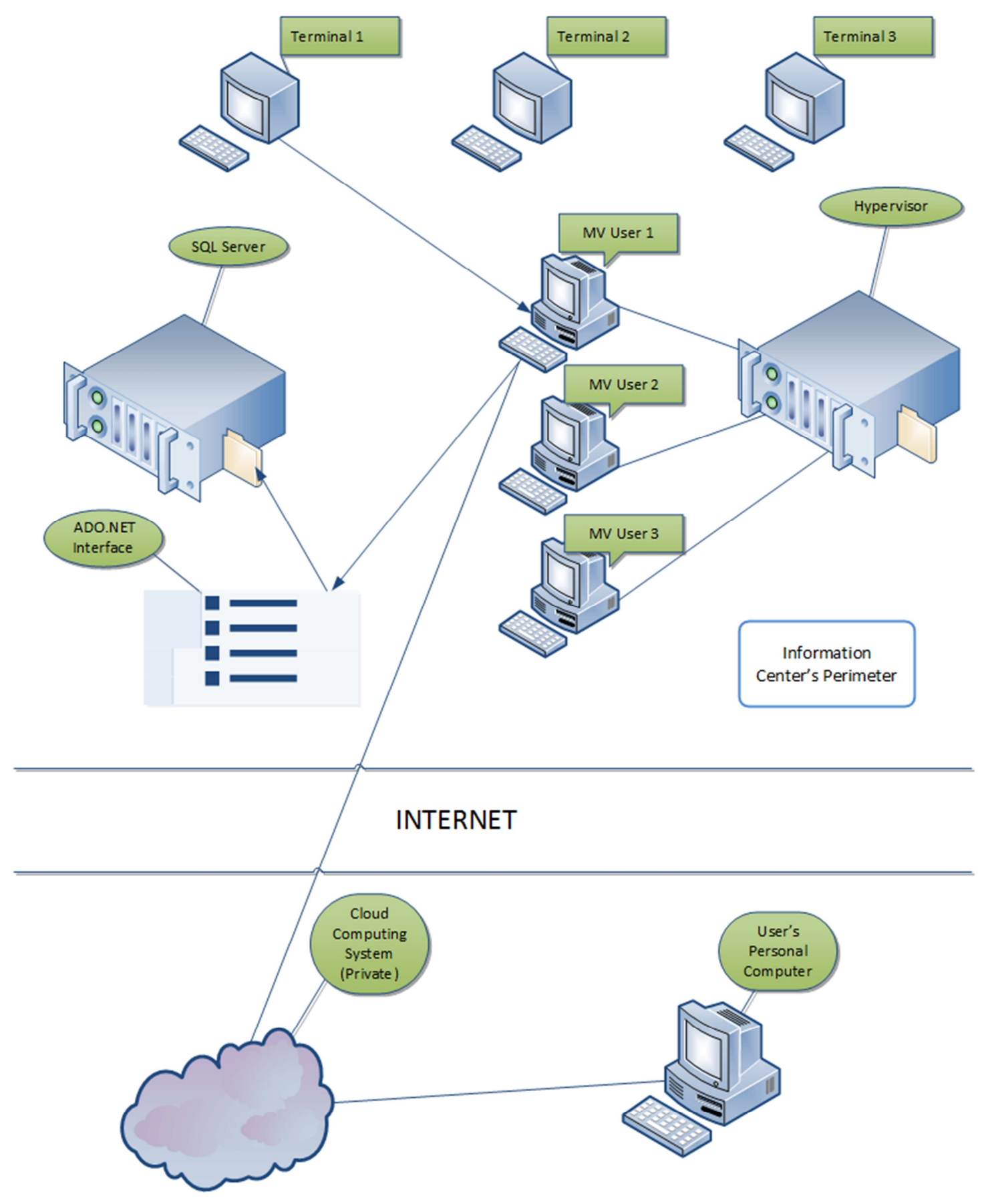

Figure 2. Framework Implementation.

\section{Conclusions}

Framework's development may allow IC as well as libraries to provide a larger amount of data in less time. Makes it unnecessary for investigators to carry their personal computers with them to IC/ Libraries, making investigation and data access more confortable. It also avoids the need of access control systems for equipment's entry into IC' $\mathrm{s}$ data access area. It allows users/ investigators to keep available data obtained in previous sessions within a private workplace, i.e., in his virtual computer. A database architecture will ensure an easy and fast digital data input on it.

\section{Acknowledgements}

I am grateful to CIDEHUS and the University of Évora for their support.

\section{References}

[1] LANKES, D. (2008). Libraries are Obsolete. OLA Quarterly, vol. 18, nr. 2 .

[2] GROSS, M. (2003). Cost, Statistics, Measures, and Standars for Digital Reference Services. Library Trends. 
[3] DARTON, R. (2008). The Research Library in Digital Age. Harvard University Library.

[4] WOODEN, R. (2006). The Future of Public Libraries in an Internet Age. Wiley Periodicals.

[5] SHERMAN, W. (2008). 33 Reasons why Libraries an Librarians are still extremely important. White Papel.

[6] MARSHALL, D. (2006). Advanced Server Virtualization. Auerbach Publications.

[7] UDDIN, M. (2011). Virtualization Implementation Model for Cost Effective Data Centers. International Journal of Ad, 69 a74.

[8] RICE, M. (2014). Windows Server 2012 R2. SYBEX.

[9] SINGH, A. (Janeiro de 2004). An Introduction to Virtualization. Obtido de Kernelthread.

[10] http://www.kernelthread.com/publications/virtualization/

[11] VIMERCATI, S. (2011). Secure Architectures in the Cloud. Dagstuhl Seminar.

[12] VMware. (s. d.). DRS and DPM. Obtido de VMware: http://www.vmware.com/products/drs
[13] VMware. (s. d.). High availability. Obtido de VMware: http://www.vmware.com/products/high-availability/

[14] VOORSLUYS, W., Broberg, J., \& Buyya, R. (2011). Introducting to Cloud Computing. Em J. Broberg, R. Buyya, \& A. Goscinski, Clound.

[15] Computing - Principles and Paradigms (p. 3). New Jersey: John Wiley \& Dons.

[16] WAHLDE, V. (1993). Digital Libraries. Association of Research Libraries.

[17] WEINBERGER, D. (2011). Too Big to Know. New York: Basic Books.

[18] WITTEN, I. (2014). Digital Libraries and Society: new perpectives on information dissemination. Hamilton: University of Waikato.

[19] YUVARAJ, M. (2014). Cloud Libraries: Issues and Chalanges. Em Cloud Computing and Virtualization Technologies in Libraries (p. 385). HERSHEY: IGI Global.

[20] MICROSOFT (2013). Microsoft Private Cloud fast Track Reference Architecture Program. 